\title{
Generation of statistical scenarios of short-term wind power production
}

\author{
Pinson, Pierre; Papaefthymiou, George; Klockl, Bernd; Nielsen, Henrik Aalborg
}

Published in:

IEEE PowerTech Conference 2007, Lausanne, Switzerland

Link to article, DOI:

10.1109/PCT.2007.4538366

Publication date:

2007

Document Version

Publisher's PDF, also known as Version of record

Link back to DTU Orbit

Citation (APA):

Pinson, P., Papaefthymiou, G., Klockl, B., \& Nielsen, H. A. (2007). Generation of statistical scenarios of shortterm wind power production. In IEEE PowerTech Conference 2007, Lausanne, Switzerland IEEE.

https://doi.org/10.1109/PCT.2007.4538366

\section{General rights}

Copyright and moral rights for the publications made accessible in the public portal are retained by the authors and/or other copyright owners and it is a condition of accessing publications that users recognise and abide by the legal requirements associated with these rights.

- Users may download and print one copy of any publication from the public portal for the purpose of private study or research.

- You may not further distribute the material or use it for any profit-making activity or commercial gain

- You may freely distribute the URL identifying the publication in the public portal

If you believe that this document breaches copyright please contact us providing details, and we will remove access to the work immediately and investigate your claim. 


\title{
Generation of Statistical Scenarios of Short-term Wind Power Production
}

\author{
Pierre Pinson, George Papaefthymiou, Member, IEEE, Bernd Klöckl, Member, IEEE, and Henrik Aa. Nielsen
}

\begin{abstract}
Short-term (up to 2-3 days ahead) probabilistic forecasts of wind power provide forecast users with a paramount information on the uncertainty of expected wind generation. Whatever the type of these probabilistic forecasts, they are produced on a per horizon basis, and hence do not inform on the development of the forecast uncertainty through forecast series. This issue is addressed here by describing a method that permits to generate statistical scenarios of wind generation that accounts for the interdependence structure of prediction errors, in plus of respecting predictive distributions of wind generation. The approach is evaluated on the test case of a multi-MW wind farm over a period of more than two years. Its interest for a large range of applications is discussed.
\end{abstract}

Index Terms - wind power, uncertainty, probabilistic forecasting, multivariate Normal variable, transformation, scenarios.

\section{INTRODUCTION}

I NCREASING the value of wind generation through the improvement of prediction systems' performance is one of the priorities in wind energy research needs for the coming years [1]. Today, most of the existing wind power prediction methods provide end-users with point forecasts [2]. The parameters of the models involved are commonly obtained with minimum least square estimation. If denoting by $p_{t+k}$ the measured power value at time $t+k, p_{t+k}$ can be seen as a realization of the random variable $P_{t+k}$. In parallel, write $\hat{p}_{t+k \mid t}$ a point forecast issued at time $t$ for lead time $t+k$, based on a model $\mathrm{M}$, its parameters $\phi_{t}$, and the information set $\Omega_{t}$ gathering the available information on the process up to time $t$. Estimating the model parameters with minimum least squares makes that $\hat{p}_{t+k \mid t}$ corresponds to the conditional expectation of $P_{t+k}$, given $\mathrm{M}, \Omega_{t}$ and $\phi_{t}$ :

$$
\hat{p}_{t+k \mid t}=\mathbb{E}\left[P_{t+k} \mid \mathbf{M}, \phi_{t}, \Omega_{t}\right]
$$

Owing to their highly variable level of accuracy, a large part of the recent research works has focused on associating uncertainty estimates to these point forecasts. They take the form of e.g. risk indices or probabilistic forecasts [3]. The latter ones are the most common and utilized in practice today. Probabilistic predictions can be either derived from meteorological ensembles [4], based on physical considerations [5], or finally produced from one of the numerous

P. Pinson and H.Aa. Nielsen are with the Informatics and Mathematical Modeling department of the Technical University of Denmark.

G. Papaefthymiou is with the Power Systems Laboratory at TU Delft, the Netherlands.

Bernd Klöckl heads the Grid Unit at the Association of Austrian Electricity Companies (VEÖ), Austria. statistical methods that have appeared in the literature, see [6][8] among others. If appropriately incorporated in decisionmaking methods, they permit to significantly increase the value of wind generation. Recent developments in that direction concentrate on e.g. dynamic reserve quantification [9], optimal operation of combined wind-hydro power plants [10] or on the design of optimal trading strategies [11].

Probabilistic forecasts are generated on a per look-ahead time basis. They do not inform on the development of the prediction errors through prediction series, since they neglect their interdependence structure. However, this information is of particular importance for many decision-making processes e.g. the economic operation of storage (or conventional generation) in combination to wind power output. In order to satisfy this additional requirement, it is proposed here to generate scenarios of short-term wind power production. These scenarios are such that they respect the (nonparametric) probabilistic forecasts for the next period, in addition to relying on the most recent information about the interdependence structure of the prediction errors.

Nonparametric probabilistic forecasts of wind generation are introduced in Section II, as well as their required and desirable properties. Then, Section III describes the method for generating statistical scenarios of wind generation from nonparametric probabilistic forecasts. It is based on the transformation of the set of random variables composing probabilistic forecast series into a single multivariate Normal variable, whose covariance matrix is tracked with recursive estimation. Results from the application of the described method are given in Section IV. Emphasis is on discussing the Gaussian assumption on the transformed random variables, on the evaluation of the probabilistic correctness of the generated scenarios, and finally on the time-varying interdependence structure of prediction errors. Concluding remarks end the paper with perspectives on applications and future developments.

\section{NONPARAMETRIC PROBABILISTIC FORECASTS OF WIND POWER}

Nonparametric probabilistic predictions may take the form of quantile, interval or density forecasts. Write $f_{t+k}$ the probability density function of $P_{t+k}$, and let $F_{t+k}$ be the related cumulative distribution function. Provided that $F_{t+k}$ is a strictly increasing function, the quantile $q_{t+k}^{(\alpha)}$ with proportion $\alpha \in[0,1]$ of the random variable $P_{t+k}$ is uniquely defined as the value $x$ such that

$$
\mathrm{P}\left(P_{t+k}<x\right)=\alpha, \quad \text { or } \quad q_{t+k}^{(\alpha)}=F_{t+k}^{-1}(\alpha)
$$


A quantile forecast $\hat{q}_{t+k \mid t}^{(\alpha)}$ with nominal proportion $\alpha$ is an estimate of $q_{t+k}^{(\alpha)}$ produced at time $t$ for lead time $t+k$, given the information set $\Omega_{t}$ at time $t$.

For most decision-making processes, a single quantile forecast is not sufficient for making an optimal decision. Instead, it is necessary to have the whole information about the random variable $P_{t+k}$ for horizons ranging from few hours to several days ahead [11]. If no assumption is made about the shape of the target distributions, a nonparametric forecast $\hat{f}_{t+k \mid t}$ of the density function of the variable of interest at lead time $t+k$ can be produced by gathering a set of $m$ quantiles forecasts

$$
\hat{f}_{t+k \mid t}=\left\{\hat{q}_{t+k \mid t}^{\left(\alpha_{i}\right)} \mid 0 \leq \alpha_{1}<\ldots<\alpha_{i}<\ldots<\alpha_{m} \leq 1\right\}
$$

that is, with chosen nominal proportions spread on the unit interval. These types of probabilistic forecasts are hereafter referred to as predictive distributions. $\hat{F}_{t+k \mid t}$ denotes the cumulative distribution function related to $\hat{f}_{t+k \mid t}$. Note that interval forecasts correspond to the specific case for which only two quantiles are quoted, and whose nominal proportions are chosen to be symmetric around the median.

A requirement for nonparametric probabilistic forecasts is that the nominal probabilities, i.e. the nominal proportions of quantile forecasts, are respected in practice. Over an evaluation set of significant size, the empirical (observed) and nominal probabilities should be as close as possible. Asymptotically, this empirical coverage should exactly equal the pre-assigned probability. That required property is commonly referred to as reliability. Besides this requirement, it is highly desirable that probabilistic predictions provide forecast users with a situation-dependent assessment of the prediction uncertainty. The shape of predictive distributions should then vary depending on various external conditions. For the example of wind power forecasting, it is intuitively expected that prediction intervals (for a given nominal coverage rate) should not have the same size when predicted wind speed equals zero and when it is near cut-off speed. This desirable property of probabilistic forecasts is commonly referred to as their sharpness of resolution. For a more thorough discussion on these various aspects, see [12], [13].

\section{GENERATING SCENARIOS OF WIND POWER PRODUCTION}

Given the nonparametric probabilistic forecasts described above, it is necessary to capture the interdependence structure of the prediction errors. For that purpose, it is proposed to take advantage of a property of reliable probabilistic predictions, which is such that the prediction errors can be made Gaussian by applying a suitable transformation. The interdependence structure of these transformed errors can then be summarized by a covariance matrix, which is recursively estimated in order to accommodate long-term variations. The main points of the method are described in the following.

\section{A. The Gaussian multivariate random variable}

Let us focus on a single look-ahead time $k$. As explained above, the predictive distributions $\left\{\hat{f}_{t+k \mid t}\right\}_{t}$ for that lookahead time are defined as reliable if the observed proportions for each of the quantiles correspond to the nominal ones [12]. In such a case, the random variable $Y_{k}$ whose realization $Y_{k}^{(t)}$ at time $t$ is defined by

$$
Y_{k}^{(t)}=\hat{F}_{t+k \mid t}\left(p_{t+k}\right), \quad \forall t
$$

is distributed uniform on the unit interval, i.e. $Y_{k} \sim \mathrm{U}[0,1]$. Consider for instance predictive distributions defined by quantiles whose nominal proportions are uniformly spread between 0 and 1 . This property intuitively translates to saying that there is the same probability the measured power value at time $t+k$ falls in any of the interval defined by two neighboring quantile forecasts $\hat{q}_{t+k \mid t}^{\left(\alpha_{i}\right)}$ and $\hat{q}_{t+k \mid t}^{\left(\alpha_{i+1}\right)}, i=1, \ldots, m-1$. Note that in practice, a continuous cumulative distribution function for each look-ahead time is obtained by fitting a smooth curve through the set of $m$ predictive quantiles.

Then, given the uniformly distributed random variable $Y_{k}$, a straightforward way to obtain a Normally distributed variable $X_{k}$ is to apply a second transformation, that uses the probit function, which corresponds to the inverse of the Gaussian cumulative distribution function. The probit function is defined as

$$
\Phi^{-1}: p \rightarrow \sqrt{2} \operatorname{erf}^{-1}(2 p-1)
$$

where ' $\operatorname{erf}^{-1}$ ' is the inverse error function. Consequently, the transformation of $Y_{k}$ to $X_{k}$ is obtained by applying the probit function to every realization $Y_{k}^{(t)}$, i.e.

$$
X_{k}^{(t)}=\Phi^{-1}\left(Y_{k}^{(t)}\right), \quad \forall t
$$

such that the random variable $X_{k}$ is distributed Gaussian with zero mean and unit standard deviation, $X_{k} \sim \mathcal{N}(0,1)$.

Considering the transformed random variables $X_{k}$ for each look ahead time, it is assumed that the random vector $\mathbf{X}=$ $\left(X_{1} X_{2} \ldots X_{K}\right)$, where $K$ is the maximum forecast horizon, follows a multivariate Normal distribution, $\mathbf{X} \sim \mathcal{N}\left(\mu_{0}, \Sigma\right)$, with the vector $\mu_{0}$ of mean values being a vector of zeros. In addition, $\Sigma$ is the covariance matrix that contains the whole information about the covariances of the random variables $X_{k}, k=1, \ldots, K$. It has 1 -values on its diagonal, since the diagonal elements give the variance of each of the random variables. Hereafter $\mathbf{X}^{(t)}$ denotes the realization of $\mathbf{X}$ at time $t$.

Note that this assumption of $\mathbf{X}$ being a multivariate Normal distribution is the simplest assumption one can make about the correlation pattern of the individual random variables $X_{k}$. More complex correlation structures could be modeled by using the copula theory, see e.g. [14]. This would not affect the other parts of the proposed method.

\section{B. Recursive estimation of the covariance matrix}

In the context of wind power forecasting applications, measurements are regularly collected and consequently used for updating the parameters of the prediction methods. A similar approach based on a recursive formulation can be applied for the adaptive estimation of the covariance matrix $\Sigma$.

Write $\Sigma_{t}$ the covariance matrix estimated from observations up to time $t$. An unbiased estimate of $\Sigma_{t}$ is commonly given 


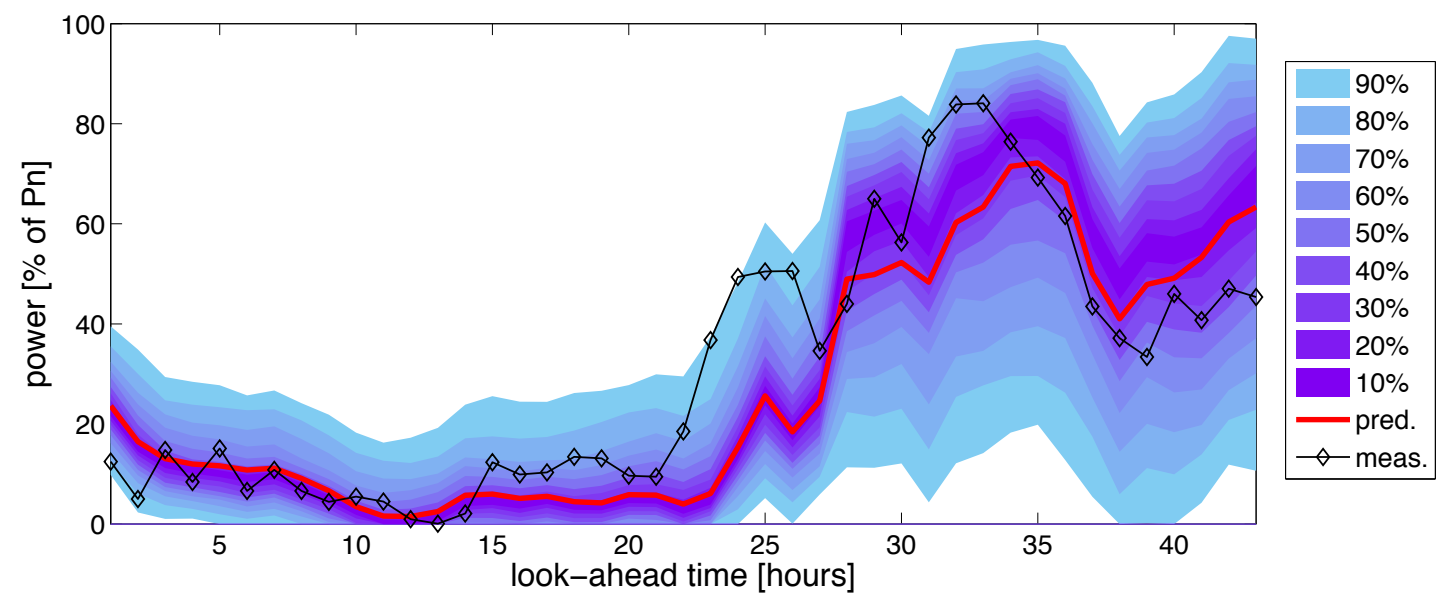

Fig. 1. Example of probabilistic predictions of wind generation in the form of nonparametric predictive distributions. Point predictions are obtained from wind forecasts and historical measurements of power production, with the WPPT method. They are then accompanied with interval forecasts produced with adaptive quantile regression. The nominal coverage rates of the prediction intervals are set to $10,20, \ldots$, and $90 \%$.

by

$$
\Sigma_{t}=\frac{1}{t-1} \sum_{t} \mathbf{X}^{(t)} \mathbf{X}^{(t)}{ }^{\top}
$$

with.$^{\top}$ the transposition operator and $\mathbf{X}^{(t)}$ the vector of observation of $\mathbf{X}$ at time $t$.

Given that the covariance estimation is based on a normalized sum, a recursive formulation for the updating of $\Sigma_{t}$ at time $t$ is readily obtained as

$$
\Sigma_{t}=\left(\frac{t-2}{t-1}\right) \Sigma_{t-1}+\left(\frac{1}{t-1}\right) \mathbf{X}^{(t)} \mathbf{X}^{(t)}{ }^{\top}
$$

Considering that the process characteristics are slowly varying leads to the application of an exponential forgetting scheme to the recursive updating formula (8). This would yield

$$
\Sigma_{t}=\lambda\left(\frac{t-2}{t-1}\right) \Sigma_{t-1}+\left(1+\lambda\left(\frac{1}{t-1}-1\right)\right) \mathbf{X}^{(t)} \mathbf{X}^{(t)}{ }^{\top}
$$

where $\lambda$ is the forgetting factor, $\lambda \in[0,1[$. This updating formula is such that when $t$ tends towards infinity, it becomes

$$
\Sigma_{t}=\lambda \Sigma_{t-1}+(1-\lambda) \mathbf{X}^{(t)} \mathbf{X}^{(t)^{\top}}
$$

which corresponds to the classical formula for exponential forgetting. The covariance matrix is initialized by setting all its off-diagonal elements to 0 and its diagonal elements to 1 .

\section{Scenario generation}

At time $t$ are available a predictive distribution $\hat{f}_{t+k \mid t}$ for each look-ahead time $k, k=1, \ldots, K$, and an estimate of the covariance matrix $\Sigma_{t}$. The procedure for obtaining a number $d$ of scenarios of wind power production for the following $K$ look-ahead times is as following:

- (i) one uses a multivariate Normal random number generator with zero mean and covariance matrix $\Sigma_{t}$ in order to have $d$ realizations of the random variable $\mathbf{X}_{t}, \mathbf{X}_{t} \sim$ $\mathcal{N}\left(\mu_{0}, \Sigma_{t}\right)$. Denote by $\mathbf{X}_{t}^{(i)}$ the $i^{\text {th }}$ of these $d$ realizations;
- (ii) $d$ realizations $Y_{k, t}^{(i)}$ of the uniform variable $Y_{k, t}$ for each look-ahead time $k$ are obtained by applying the inverse probit function $\Phi$ to each component of $\mathbf{X}_{t}^{(i)}$ :

$$
Y_{k, t}^{(i)}=\Phi\left(X_{k, t}^{(i)}\right), \quad \forall k, i
$$

- (iii) the scenarios of wind power production finally result from the application, for each look-ahead time $k$, of the inverse cumulative distribution function $\hat{F}_{t+k \mid t}^{-1}$ to the $d$ realizations $Y_{k, t}^{(i)}$ of $Y_{k, t}$ for that look-ahead time:

$$
\hat{p}_{t+k \mid t}^{(i)}=\hat{F}_{t+k \mid t}^{-1}\left(Y_{k, t}^{(i)}\right), \quad \forall k, i
$$

\section{Results}

Focus is given in this Section to the application of the method to a real-world test case, in order to illustrate its qualities. After describing the case-study considered, we verify that transformed variables are indeed close to being Gaussian, and that the generated scenarios are probabilistically correct, i.e. that they respect the probabilistic forecasts used as input. Finally, the evolution of the interdependence structure of forecast errors through time is discussed.

\section{A. Description of the case-study}

Predictions are produced for a Danish onshore wind farm with a nominal capacity $P_{n}$ of $21 \mathrm{MW}$. The period for which both predictions and related power measures are available goes from March 2001 until end of April 2003. The point predictions result from the application of the WPPT method [15], which uses meteorological predictions of wind speed and direction as input, as well as historical measurements of power production. These point predictions have an hourly resolution up to 43-hour ahead, and are updated every hour. All predictions and measures are normalized by $P_{n}$. The dataset includes 16900 point prediction series.

Nonparametric probabilistic forecasts are produced with adaptive quantile regression [7]. Predictive distributions are 


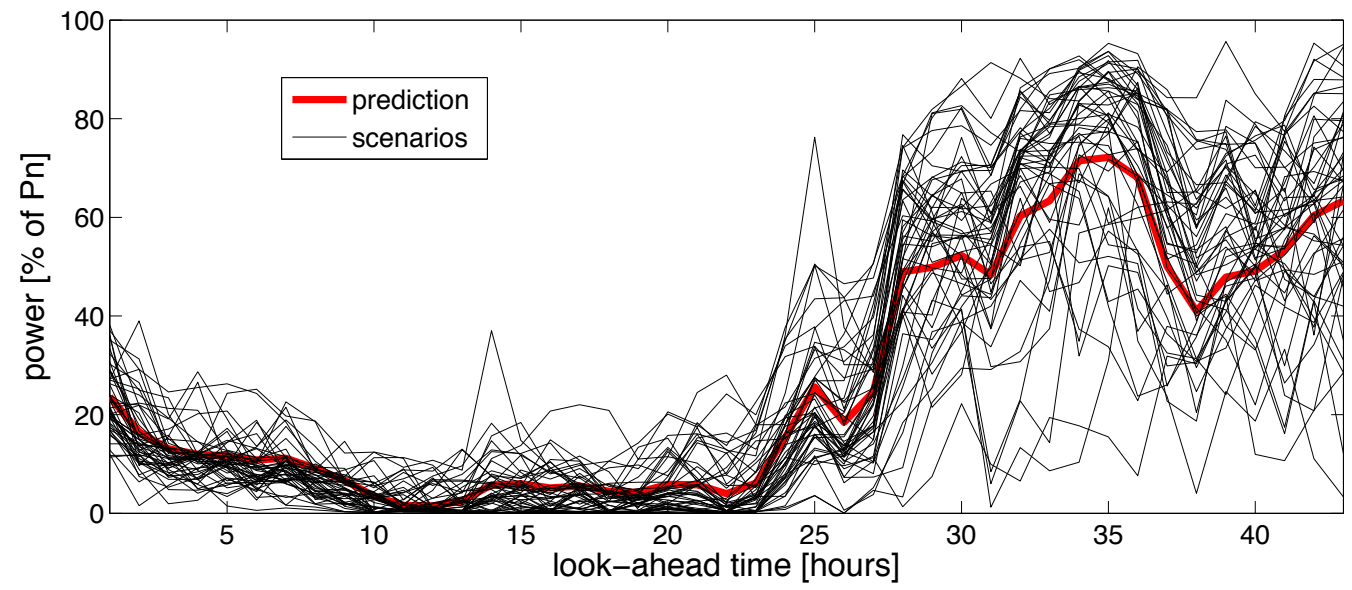

Fig. 2. Example of wind power point predictions with 50 alternative scenarios produced from the method described in the paper (for the same period as in Fig. 1). The point prediction series correspond to the most likely scenario while the others reflect the prediction uncertainty and the interdependence structure of predictions errors.

given by 19 quantile forecasts whose nominal proportions range from 0.05 to $0.95 \%$ by 0.05 increments. From a probabilistic point of view, since it is not possible to exclude any possibility, the predictive quantiles with nominal proportions 0 and 1 are always set to normalized power values of 0 and 1 , respectively, whatever the look-ahead time. The quality of this method is evaluated and discussed in [12]. It has been shown to have an acceptable level of reliability, and a superior overall skill when compared with other nonparametric probabilistic forecasting methods of the state of the art.

Fig. 1 gives an example of such probabilistic forecasts of wind generation, in the form of a fan chart. In parallel, Fig. 2 depicts a set of 50 scenarios of power production for the same period, generated from the method introduced, along with the traditionally provided point forecasts for the coming period. For this illustration only 50 scenarios are produced, but since the method is not highly computationally expensive, one can raise this number to several thousands in order to be used in e.g. monte-carlo simulations for decision-making. For the following evaluation, the number of scenarios is set to 10000 . The second parameter of the method is the forgetting factor $\lambda$ that permits an adaptive tracking of the interdependence structure of prediction errors. It is set here to 0.995, which corresponds to an effective number of 200 observations.

\section{B. Gaussian assumption on transformed random variable}

The assumption such that the transformed random variable $X_{k}$ is Gaussian is discussed here. For that, the realizations $X_{k}^{(t)}$ of $X_{k}$ are collected over the whole dataset. In principle, one would use a test of Normality for verifying that such an assumption is acceptable. Actually, this kind of test could also be used for verifying the reliability of nonparametric probabilistic forecasts. For literature on Normality testing, we refer to [16] and references therein.

For discussing the Normality of the transformed random variables, the distributions of these realizations are compared to the probability distribution function (pdf) of a $\mathcal{N}(0,1)$ distribution. Fig. 3 gives the example of the common plotting of these two distributions, for the case of $k=18$ i.e. for the transformed random variable related to 18 -hour ahead forecasts. The distribution of observations appears to be slightly left-skewed and sharper than the pdf of a $\mathcal{N}(0,1)$ distribution, though they look very similar.

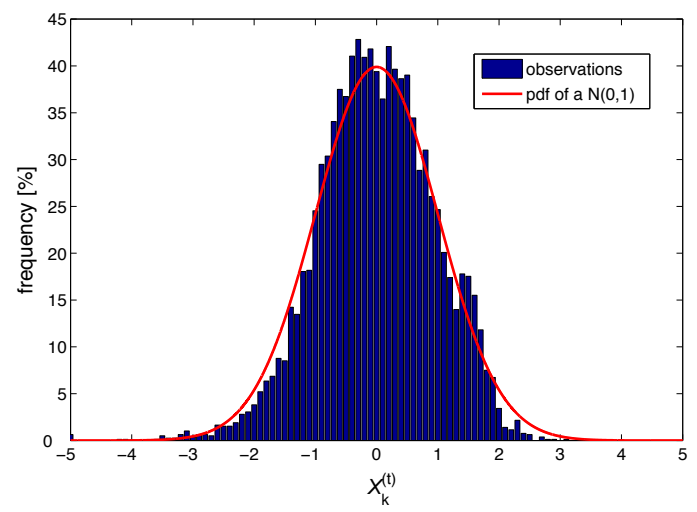

Fig. 3. Visual comparison between the set of observations $\left\{X_{k}^{(t)}\right\}$ (for $k=18$, i.e. for the transformed random variable related to 18 -hour ahead prediction) and the probability distribution function of a $\mathcal{N}(0,1)$ distribution.

The four moments of the distributions of these realizations, i.e. their mean $\mu_{X}(k)$, their standard deviation $\sigma_{X}(k)$, their skewness $\gamma_{X}(k)$ and finally their excess kurtosis $\kappa_{X}(k)$, are calculated for each of the 43 transformed random variables corresponding to each look-ahead time $k$. Their average value over the set of look-ahead times and related standard deviation, denoted by - and $\sigma($.$) respectively, are gathered in Table I.$

Whatever $k$, the distributions are slightly left-skewed, as it was observed for the example of $k=18$ in Fig. 3. In addition, the excess kurtosis is positive, with low values though, indi- 
TABLE I

SUMMARY STATISTICS RELATED TO THE MOMENTS OF THE DISTRIBUTIONS OF THE OBSERVATIONS OF THE TRANSFORMED RANDOM VARIABLES $X_{k}$

\begin{tabular}{cccc}
\hline \hline $\bar{\mu}_{X}$ & $\bar{\sigma}_{X}$ & $\bar{\gamma}_{X}$ & $\bar{\kappa}_{X}$ \\
0.0018 & 0.95 & -0.20 & 0.36 \\
\hline$\sigma\left(\mu_{X}\right)$ & $\sigma\left(\sigma_{X}\right)$ & $\sigma\left(\gamma_{X}\right)$ & $\sigma\left(\kappa_{X}\right)$ \\
0.0015 & 0.01 & 0.05 & 0.24 \\
\hline \hline
\end{tabular}

cating that $X_{k}$-observations distributions are sharper in their central part, and have longer tails, than a $\mathcal{N}(0,1)$. These slight deviations from a perfect Normal shape are due to the fact that the nonparametric predictions are not perfectly reliable in the low power range [12]. Regarding the first two moments, one sees that transformed variables are clearly centered with a standard deviation close to 1 .

\section{Probabilistic correctness of generated scenarios}

In a second part, it is verified if the generated scenarios are probabilistically correct, i.e. if they respect the marginal distributions of the probabilistic forecasts used as input. For this evaluation, we use the fact that the proportion of generated scenarios that lies between two successive quantiles of a given predictive distribution should correspond to the difference in nominal probability for these two quantiles. For instance, for a given predictive distribution, $5 \%$ of generated scenarios should lie in the interval formed by the quantiles with nominal proportions 0.4 and 0.45 . Such an evaluation can be summarized in a Probability Integral Transform (PIT) histogram, as introduced in e.g. [13]. The PIT histogram of Fig. 4 summarizes this evaluation over the whole dataset and for all horizons, since it has been witnessed that the probabilistic correctness was not significantly different for the various look-ahead times.

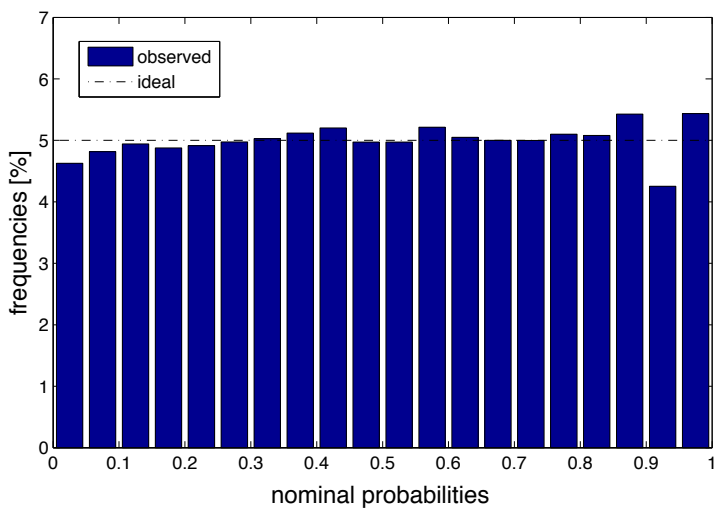

Fig. 4. Evaluation of the probabilistic correctness of generated scenarios with a PIT histogram. The ideal case of perfect probabilistic correctness is represented by the dash-dot line. This PIT histogram gathers the results for all look-ahead times.

The ideal situation corresponds to the case for which each bin would contain $5 \%$ of the scenarios. The deviations from this idea situations are low here, indicating that the generated scenarios have distributions similar to that of the predictive distributions, and this all over the range of nominal probabilities.

\section{On the tracking of the covariance matrix}

In a last stage, we follow the evolution of the covariance matrix of the multivariate Normal variable $\mathbf{X}$ through the dataset. Remember that through suitable transformation, this covariance matrix describes the interdependence structure of prediction errors for the various look-ahead times. Figure 5 proposes a visualization of the elements of $\Sigma_{t}$ for two points in time of the dataset considered, i.e. $t=300$ hours and $t=2500$ hours. The $x-$ and $y$-axis are for the prediction horizon $k$.

The maximum values of the elements of $\Sigma_{t}$ lie on the diagonal. They correspond to the variances of the marginal distributions of prediction errors for each prediction horizon. They are close to 1 . The covariance values sharply decrease as the difference between prediction horizons increases. Though, one notices that the covariance patterns differ for the two points in time. In the first case ( $t=300$ hours), there is a significantly stronger covariance pattern, with more specifically a high correlation between prediction errors for horizons between 12 and 32-hour ahead. Such phenomenon may relate to the point prediction method not capturing a diurnal component of the wind generation at this site. This covariance pattern is not present in the second case, i.e. for $t=2500$ hours. This demonstrates the interest of the adaptive tracking of $\Sigma_{t}$.

\section{Conclusions And Perspectives}

Probabilistic forecasts of wind generation are a highly valuable input to a number of decision-making problems related the management or trading of wind generation. However, the fact that they do not provide any information on the interdependence structure of prediction errors make them almost useless for a large class of decision-making problems for which successive decisions are interdependent. This concerns for instance the optimal operation of wind in combination with storage or conventional generation, or the design of trading strategies for the participation in various markets with different gate closures. In the present paper, we have described a method that permits to generate scenarios of wind generation that respect both the predictive distributions and the interdependence structure of prediction errors.

The various qualities of the method have been illustrated for the test case of a multi-MW wind farm, for which both point predictions and nonparametric probabilistic forecasts were obtained from a state-of-the-art method. Future developments related to the method for generating scenarios will focus on the covariance matrix of the transformed random variable. More specifically, it is envisaged to: (i) propose a method for an optimal choice of the forgetting factor; (ii) make the covariance matrix a function of influential variables, in order to account for their impact on the interdependence structure of prediction errors; (iii) extend the method in order to account for the spatio-temporal aspects of this interdependence structure.

Broader perspectives relate to the use of wind generation scenarios in a range of decision-making problems, and to the verification of the resulting benefits. 

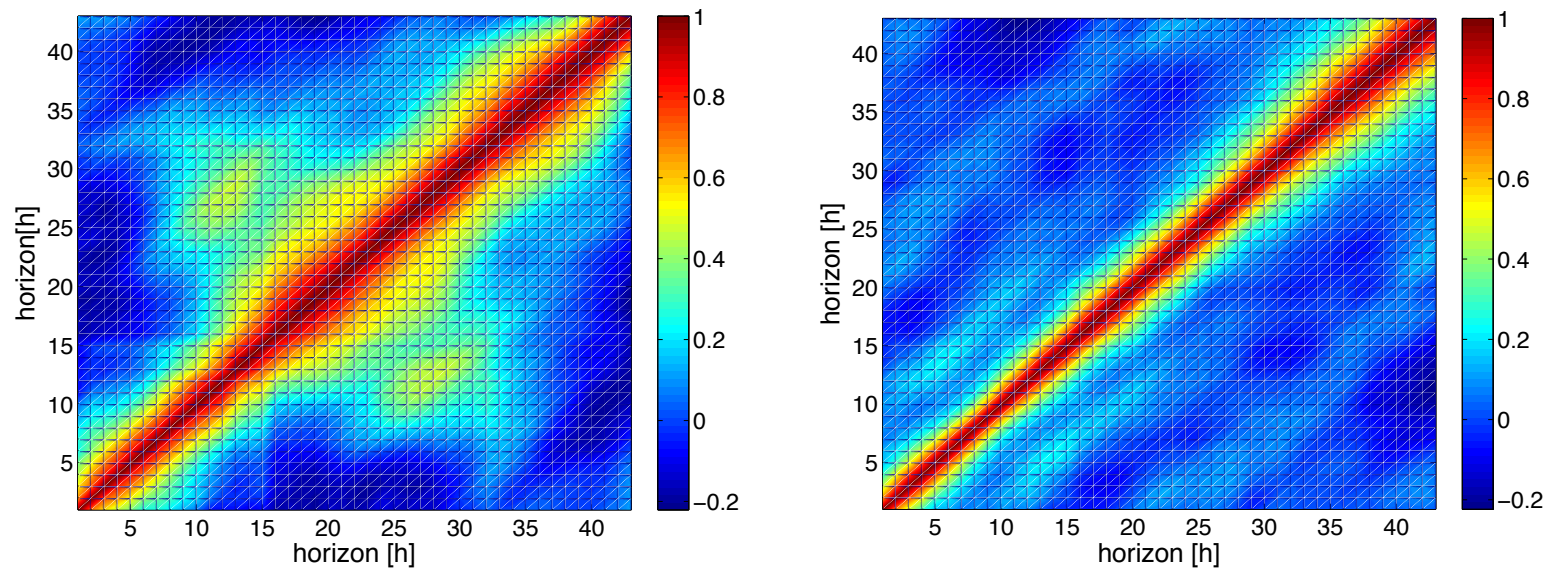

Fig. 5. Visualization of the covariance matrices of the multivariate normal random variable that permit to estimate and track the interdependence structure of prediction errors. Plots are for two time points of the dataset: after 300 hours (left) and after 2500 hours (right).

\section{ACKNOWLEDGMENTS}

The authors greatly acknowledge Dong Energy and Vattenfall Denmark for providing the power data used in the present paper.

\section{REFERENCES}

11] S.-E. Thor and P. Weis-Taylor, "Long-term research and development needs for wind energy for the time frame 2000-2020," Wind Energ., vol. 5, pp. 73-75, 2003.

[2] G. Giebel, G. Kariniotakis, and R. Brownsword, "State of the art on short-term wind power prediction," 2003, ANEMOS Deliverable Report D1.1, available online: http://anemos.cma.fr.

[3] P. Pinson and G. Kariniotakis, "On-line assessment of prediction risk for wind power production forecasts," Wind Energ., vol. 7, no. 2, pp. 119-132, 2004

[4] H. A. Nielsen, T. S. Nielsen, H. Madsen, J. Badger, G. Giebel, L. Landberg, K. Sattler, L. Voulund, and J. Tøfting, "From wind ensembles to probabilistic information about future wind power production - results from an actual application," in Proc. IEEE PMAPS 2006, Stockholm, Sweden, 2006.

[5] M. Lange and U. Focken, Physical Approach to Short-Term Wind Power Prediction. Springer, 2005.

[6] T. Gneiting, K. Larson, K. Westrick, M. G. Genton, and E. Aldrich, "Calibrated probabilistic forecasting at the stateline wind energy center: The regime-switching space-time method," J. Am. Stat. Assoc., vol. 101, no. 475, pp. 968-979, 2006, Applications and Case-studies.

[7] J. K. Møller, H. A. Nielsen, and H. Madsen, "Time adaptive quantile regression," Comp. Stat. Data Anal., 2006, submitted.

[8] P. Pinson, "Estimation of the uncertainty in wind power forecasting," Ph.D. dissertation, Ecole des Mines de Paris, Paris, France, 2006.

[9] R. Doherty and M. O'Malley, "A new approach to quantify reserve demand in systems with significant installed wind capacity," IEEE Trans. Power Syst., vol. 20, no. 2, pp. 587-595, 2005.

[10] E. D. Castronuovo and J. A. Pecas Lopes, "On the optimization of the daily operation of a wind-hydro power plant," IEEE Trans. Power Syst., vol. 19, no. 3, pp. 1599-1606, 2004.

[11] P. Pinson, C. Chevallier, and G. Kariniotakis, "Trading wind generation with short-term probabilistic forecasts of wind power," IEEE Trans. Power Syst., 2006, forthcoming.

[12] P. Pinson, H. A. Nielsen, J. K. Møller, H. Madsen, and G. Kariniotakis, "Nonparametric probabilistic forecasts of wind power: required properties and evaluation," Wind Energ., 2007, forthcoming.

[13] T. Gneiting, F. Balabdaoui, and A. E. Raftery, "Probabilistic forecasts, calibration and sharpness," J. R. Statist. Soc. B, vol. 69, pp. 243-268, 2007.

[14] G. Papaefthymiou, "Integration of stochastic generation in power systems," Ph.D. dissertation, Delft University of Technology, Delft, The Netherlands, 2007.
[15] T. S. Nielsen, H. Madsen, and H. A. Nielsen, "Prediction of wind power using time-varying coefficient functions," in Proc. IFAC 2002, Barcelona, Spain, 2002.

[16] I. A. Ahmad and A. R. Mugdadi, "Testing normality using kernel methods," J. Nonparametr. Stat., vol. 15, pp. 273-288, 2003.

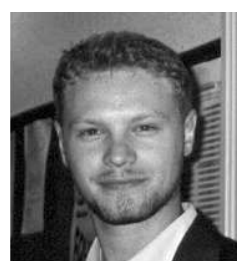

Pierre Pinson received the M.Sc. degree in Applied Mathematics from the National Institute for Applied Sciences (INSA Toulouse, France) in 2002 and the $\mathrm{Ph} . \mathrm{D}$. degree in Energetic from Ecole des Mines de Paris in 2006. He is currently with the Informatics and Mathematical Modeling department of the Technical University of Denmark as an Assistant Professor. His research interests include among others forecasting, uncertainty estimation, optimization under uncertainty, decision sciences, and renewable energies.

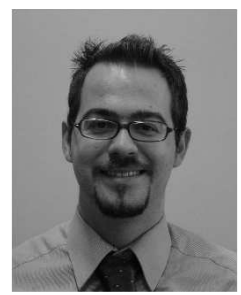

George Papaefthymiou obtained his Dipl-Eng in Electrical and Computer Engineering from the University of Patras, Greece in 1999. He is currently pursuing Ph.D. at the Electrical Power Systems Laboratory of the Department of Electrical Engineering, Mathematics and Computer Science of the Delft University of Technology, in the main framework of Intelligent Power Systems. His current research interests include modeling of uncertainty in power systems and design of systems with large-scale penetration of distributed and stochastic generation.

Bernd Klöckl received his MSc degree from Graz University of Technology, Austria, in the areas of electric machines and high voltage technology in 2001. Between 2002 and 2006, he was with the High Voltage Laboratory of ETH Zurich, Switzerland. He now heads the Grid Unit at the Association of Austrian Electricity Companies (VEÖ), Austria. He is a Member of the IEEE.

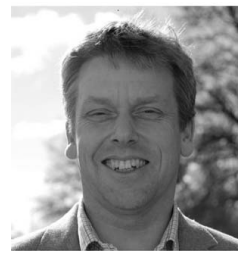

Henrik Aa. Nielsen received the M.Sc. degree in engineering from the Technical University of Denmark in 1991, where he studied energy systems, optimization, and statistics. He worked as statistician in the pharmaceutical industry from 1991 to 1994 and received the Ph.D. Degree in Statistics in 2000. $\mathrm{He}$ has been working extensively with time-series data originating from energy systems, with focus on modelling and forecasting. His research interests are related to non- and semi-parametric methods with applications to time-series data. 